\title{
Eintrittsgeld für Artenvielfalt
}

\section{Die Property-Rights-Theorie liefert Ansäłze, um die biologische Vielfalt der Erde zu bewahren. Wenn die Ursprungsländer durch klar zugewiesene Verfö- gungsrechfe Erträge aus den genetischen Ressourcen ziehen können, bekommen sie auch einen Anreiz, in den Erhalt dieser Ressourcen zu investieren.}

I

Von Achim Lerch

n Übereinstimmung mit einer zentralen Aus-

sage der ökonomischen Theorie der Verfügungsrechte, wonach immer dann ein Anreiz zur Definition und Durchsetzung von Eigentumsrechten an einer Ressource entsteht, wenn der damit verbundene Nutzen die Kosten übersteigt, ist seit einigen Jahren die Etablierung von Eigentumsrechten an genetischen Ressourcen zu beobachten. Sowohl in internationalen Verhandlungen als auch im Rahmen von Verträgen zwischen Ursprungsländern und interessierten Unternehmen aus der Pharma-, Saatgut- oder Kosmetikbranche wird der Zugang zu diesen Ressourcen zum Teil neu geregelt. Andererseits gibt es starke Anhaltspunkte dafïr, daß - wiederum in Übereinstimmung mit den Vorhersagen der ökonomischen Theorie - bislang fehlende oder unzureichend spezifizierte Eigentumsrechte an biologischen Ressourcen mit zu der beobachteten weltweiten Gefährdung der Artenvielfalt beitragen.

\section{- Die ambivalente Formel vom gemeinsamen Menschheitserbe}

Stellt man zunächst die grundsätzliche Frage, wem die Eigentumsrechte an den biologischen Ressourcen zukommen - wem die biologische Vielfalt des Planeten ,gehört" -, so lautet die Antwort häufig, daß es sich hierbei um das "gemeinsame Erbe der Menschheit" handle. Die Schwierigkeiten, die mit diesem wohlklingenden Begriff verbunden sind, zeigen sich jedoch bei genauerem Hinsehen. Der Gedanke des "gemeinsamen Menschheitserbes" enthält zwei Elemente, die zunächst getrennt werden sollten. Zum einen das Prinzip der Gemeinsamkeit, das heißt, des gemeinsamen Zugangs für alle, zum anderen den Gedanken des Erbes, und das heißt . auch des Vererbens an nachfolgende Generationen, den Gedanken des Bewahrens.

Beachtenswert ist, daß sich beide Elemente bei einer oberflächlichen Interpretation unter gewissen Umständen gegenseitig ausschließen können. Wenn etwa der gemeinsame Zugang zur „common heritage“ ausdrücklich mit der „Abwesenheit von Eigentum“ gleichgesetzt wird, also vom freien, unbeschränkten Zugang für alle ausgegangen wird, so ist damit ja gerade eine Open-access-Situation beschrieben, die bekanntermaßen starke Anreize zur Übernutzung impliziert. Dies ist aber unvereinbar mit der Forderung nach Bewahrung und Vererbung an nachfolgende Generationen. Soll also das gemeinsame Menschheitserbe als funktionierendes Gemeineigentum verstanden werden, so bedeutet es gerade nicht die Abwesenheit von Eigentum, sondern erfordert die klare Zuweisung von möglichst genau spezifizierten Verfiigungsrechten. Ein ,gemeinsames Menschheitserbe" allein entbindet also nicht von der Notwendigkeit, genau festzulegen, wer in welchem Maße an diesem gemeinsamen Erbe partizipieren darf.

Mit der Formel des gemeinsamen Menschheitserbes und unter Verweis auf die Kollektivguteigenschaften haben in der Vergangenheit vor allem die Nachfrager nach biologischen Ressourcen in den Industrieländern den freien Zugang zu und die kompensationslose Entnahme von Pflanzen und Tieren in den artenreichen Ländern des Südens gerechtfertigt. In dieser Argumentation wurde einerseits das positive Merkmal öffentlicher Güter, die Nichtausschließbarkeit, ins Normative gewendet: Aus der Tatsache, daß niemand von der Nutzung eines Kollektivgutes ausgeschlossen werden kann, wurde gefolgert, daß auch niemand ausgeschlossen werden solle. Aus allokationstheoretischer Sicht liegt hierin jedoch eine Verwechslung öffentlicher mit meritorischen Gütern. Andererseits wurde das Merkmal der Nichtrivalität ganz nach dem Argumentationsmuster des "Freifahrers" als Vorwand benutzt für die Weigerung, sich an der Erstellung des Kollektivgutes zu beteiligen. Auch dies kann natürlich aus allokationstheoretischer Sicht nicht überzeugen, da mit derartigem Freifahrerverhalten volkswirtschaftliche Wohlfahrtsverluste verbunden sind. Die Ursprungsländer biologischer Vielfalt stehen denn auch dem Konzept des "gemeinsamen Menschheitserbes" eher ablehnend gegenüber, erkannten insbesondere einen Widerspruch darin, daß ihre biologischen Ressourcen das frei zugängliche gemeinsame Erbe darstellen sollen, sie aber für daraus entwickeltes Saatgut oder Medikamente bezahlen müssen. Nicht zuletzt aus diesem Grund ist dann auch der Begriff des ,gemeinsamen Menschheitserbes" nicht in die Konvention zum Schutz der biologischen Vielfalt eingegangen, die auf dem Umweltgipfel in Rio de Janeiro 1992 verabschiedet wurde. Die Konvention geht vielmehr davon aus, daß grundsätzlich die jeweiligen Ursprungsländer Verfügungsrechte über ihre biologischen Ressourcen besitzen. Gleichwohl spricht die Konvention vom Artenschutz als gemeinsamem Anliegen der Menschheit (,,common concern of humankind"). Aus dem Recht der Ursprungsländer, Erträge aus ihren genetischen Ressourcen zu ziehen, folgt eben weder, daß ihnen auch das Recht auf Vernichtung dieser Ressourcen zukommt, noch, daß ihnen alle Anstrengungen zum Artenschutz allein aufgebürdet werden sollen. Die biologische Vielfalt weist vielfach die Eigenschaften eines globalen öffentlichen Gutes auf, für das weder eine vollständige marktmäßige Bewertung noch eine lückenlose Ausstattung mit Verfügungsrechten möglich erscheint. Daher bleibt grundsätzlich die Notwendigkeit bestehen, all jene zur Finanzierung dieses Kollektivgutes heranzuziehen, die in irgendeiner Form Nutzen daraus ziehen.

\section{Theorie und Praxis sprechen für lokale Verfügungsrechte}

In dem Maße, in dem Ursprungsländer biologischer Vielfalt durch entsprechende Verfïgungsrechte in die Lage versetzt werden, Erträge aus diesen genetischen Ressourcen zu ziehen, steigt auch ihr Anreiz, in den Erhalt dieser Ressourcen zu investieren. Würde beispielsweise Madagaskar eine Beteiligung an den Gewinnen aus dem Verkauf der Krebsmedikamente erhalten, die aus dem Madagaskar-Immergrïn (Catharanthus roseus) entwickelt wurden und mit denen ein jährlicher Umsatz von weit über 100 Millionen Dollar erzielt wird, wäre dies eine bedeutende Einnahmequelle für das Land.

Gerade das Beispiel Madagaskar-Immergrün verweist auch auf einen weiteren wichtigen Aspekt: die Rechte der Bevölkerung vor Ort, sei- 
en es Farmer, die traditionell Saatgut kultivieren, oder indigene Medizinmänner mit ihrem Wissen um die Heilkraft von Pflanzen. So spielt gerade bei der Suche nach neuen medizinischen Wirkstoffen die ethnobotanische Methode eine wesentliche Rolle, und auch die Wirksamkeit des Madagaskar-Immergrüns gegen Krebs wurde nicht zufällig, sondern nach einer systematischen Untersuchung der von einheimischen „Medizinmännern“ verwendeten Heilpflanzen entdeckt. Zudem sind zahlreiche Beispiele dokumentiert, die zeigen, daß erst die Außerkraftsetzung traditioneller kommunaler und lokaler Eigentumsrechte zu gravierenden Übernutzungen geführt hat, und das U.S. National Resource Council stellte 1992 fest, darin liege die wahre „Tragödie des Gemeineigentums“.

Die Erkenntnis, daß die Außerkraftsetzung traditionellen, lokalen (Gemein-)Eigentums neben der Zerschlagung sozio-kultureller Systeme auch zur Bedrohung der biologischen Vielfalt beiträgt, fuihrt mittlerweile zunehmend zu der Einsicht, daß umgekehrt die Stärkung beziehungsweise Wiederzuweisung solcher lokaler Verfïgungsrechte zum Schutz der Biodiversität beitragen kann. Diese Sicht sieht sich wiederum im Einklang mit den zentralen Aussagen der Property-Rights-Theorie, wonach das Prinzip der Dezentralität maßgeblich ist für die Effizienz von Eigentumsrechtsgestaltungen. Aus diesen Überlegungen folgt demnach, daß die grundsätzlich anzuerkennenden nationalen Verfügungsrechte der konkreten Ausgestaltung auf lokaler/kommunaler Ebene bedürfen. Nationales Patrimonium heißt also nicht, daß die konkreten Verfügungsrechte vollständig bei der Zentralregierung angesiedelt sein müssen.

\section{Das Beispiel Costa Rica}

Auf welche Art und Weise Länder oder lokale Gemeinschaften von genetischen Ressourcen profitieren können, wenn ihre Verfügungsrechte an diesen Ressourcen anerkannt werden, zeigt das Beispiel der bilateralen Verträge zwischen Ursprungsländern biologischer Vielfalt (beziehungsweise lokalen Gemeinschaften innerhalb dieser Länder) und interessierten Unternehmen. Das bekannteste derartiger Abkommen dürfte das zwischen dem amerikanischen Pharmakonzern Merck \& Co. und dem Institut für biologische Vielfalt (INBio) in Costa Rica darstellen, das im Jahr 1991 geschlossen wurde. Der Pharmakonzern zahlt jeweils für einen Zweijahreszeitraum insgesamt 1.135.000 Dollar und erhält im Gegenzug eine (unbekannte) Anzahl an Proben von Pflanzen und Insekten aus den Nationalparks Costa Ricas. Sollte Merck aus diesen Proben marktfähige Medikamente entwickeln, erhält INBio Gewinnbeteiligungen aus dem entsprechenden Erlös. INBio ist eine private Non-Profit-Organisation, deren Hauptaufgabe in der Inventarisierung der biologischen Vielfalt Costa Ricas besteht und das zu diesem Zweck mit dem „Ministerium für nationale Ressourcen, Energie und Minen" einen Kooperationsvertrag abgeschlossen hat. Im Gegenzug für die Erlaubnis zur Probensammlung in den Nationalparks des Landes erhält das Ministerium 100.000 Dollar der 1,135 Millionen sowie 50 Prozent aller Gewinnbeteiligungen.

In der öffentlichen Diskussion über den MerckINBio-Vertrag wurde insbesondere kritisiert, daß Merck eine zu starke Exklusivität für zuwenig Geld eingeräumt würde. Diese Exklusivität ist allerdings einerseits befristet und andererseits eingeschränkt, da neben INBio auch andere Unternehmen Probensammlungen in Costa Ricas Nationalparks betreiben. Nur sehr schwer zu beantworten ist die Frage, inwieweit die von Merck gezahlte Summe einen angemessenen Preis für die erbrachte Leistung darstellt, oder ob hier, wie Kritiker meinen, Costa Ricas biologische Vielfalt „,unter Wert verschleudert" wird. $\mathrm{Zu}$ beachten ist dabei, daß diese Zahlung zunächst nur das „Eintrittsgeld“ für den Zugang zu den potentiellen genetischen Ressourcen des Landes darstellt und im Falle einer verwertbaren Entdeckung Gewinnbeteiligungen hinzukommen, deren Höhe jedoch nur den Vertragspartnern bekannt ist. Vermutungen reichen hier von einem bis 40 Prozent. Realistisch dürfte ein Wert um fünf Prozent sein.

Aus ökonomischer Sicht besteht bei derartigen Bioprospectingabkommen grundsätzlich das Problem, den ,optimalen Mix“ zwischen „Eintrittsgeld" und Gewinnbeteiligung zu ermitteln. Hohe „Eintrittsgelder" setzen eine relativ hohe Exklusivität des Zutritts voraus. Der Vorteil besteht dann in der sicheren Realisierung eines gegenwärtigen Ertrages gegenüber relativ unsicheren zukünftigen Gewinnbeteiligungen. Wird hingegen mehreren Firmen Zugang gewährt (bei geringeren „Eintrittsgeldern“ aufgrund geringerer Exklusivität), steigen durch erhöhten Wettbewerb und breiter gestreute Forschung die Chancen auf Entdeckung nützlicher Substanzen und damit auch der Erwartungswert zukünftiger Gewinnbeteiligungen.

\section{Optimale Allokation der Ressourcen nicht möglich}

Aus ökonomischer Sicht scheint es naheliegend, bilaterale Verträge wie den beschriebenen als ein Beispiel für Coase-Verhandlungen anzusehen und unter dem Blickwinkel des CoaseTheorems zu analysieren, wonach Verhandlungen zwischen den von externen Effekten betroffenen Parteien bei Abwesenheit von Transaktionskosten zu einer Pareto-optimalen Internalisierung fuihren. Abgesehen von der Tatsache, daß die Transaktionskosten im vorliegenden Fall natürlich nicht Null betragen und daß durch mögliche Informationsasymmetrien oder die ungleiche Anfangsausstattung der Verhandlungspartner weitere Probleme hinzukommen, ergibt sich ein entscheidender Einwand gegen die Effizienzvermutung des Coase-Theorems bereits aus der Tatsache, daß nicht alle von den Externalitäten Betroffenen an den Verhandlungen beteiligt sind - und im Falle zukünftiger Generationen auch nicht sein können. Diese sind aufgrund der Irreversibilität von Verlusten der Artenvielfalt direkt betroffen, ohne ihre Interessen im gegenwärtigen Verhandlungsprozeß vertreten zu können.

Die Hoffnung, die Zuweisung von klar definierten Verfügungsrechten allein würde ausreichen, um über das Instrument der Coase-Verhandlungen die Pareto-optimale Allokation biologischer Ressourcen zu erreichen, erweist sich außerhalb der Modellwelt als unerfïllbar. Dies bedeutet allerdings nicht, daß durch bilaterale Verhandlungen wie die beschriebenen nicht spürbare Pareto-Verbesserungen gegenüber dem Status quo erreicht werden könnten.

\section{Anmerkung}

Zu einer ausführlichen Darstellung und umfangreichen Literaturangaben siehe Lerch, A. (1996): Verfügungsrechte und biologische Vielfalt. Eine Anwendung der ökonomischen Analyse der Eigentumsrechte auf die spezifischen Probleme genetischer Ressourcen, Marburg: Metropolis-Verlag.

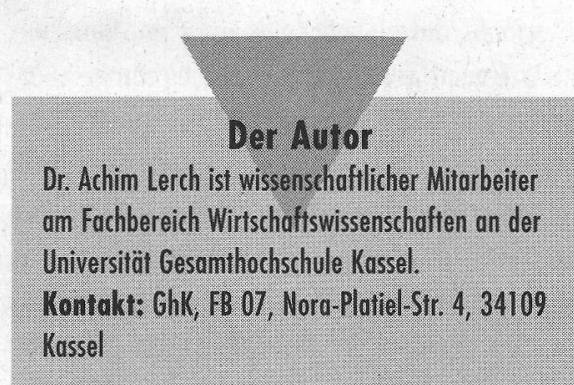


(c) 20I0 Authors; licensee IÖW and oekom verlag. This is an article distributed under the terms of the Creative Commons Attribution Non-Commercial No Derivates License (http://creativecommons.org/licenses/by-nc-nd/3.o/), which permits unrestricted use, distribution, and reproduction in any medium, provided the original work is properly cited. 